\title{
Nuclear polarization contribution to the Lamb shift in heavy atoms
}

\author{
Günter Plunien, Berndt Müller, and Walter Greiner \\ Institut für Theoretische Physik der Johann-Wolfgang-Goethe-Universität, Robert-Mayer-Strasse 8-10, Postfach 111932 , \\ D-6000 Frankfurt am Main, Federal Republic of Germany \\ Gerhard Soff \\ Gesellschaft für Schwerionenforschung, Planckstrasse 1, Postfach 110 552, \\ D-6100 Darmstadt, Federal Republic of Germany \\ (Received 8 February 1989)
}

\begin{abstract}
The energy shift of the $1 s_{1 / 2}$ state in ${ }_{92}^{238} \mathrm{U}$ due to virtual excitation of nuclear rotational modes is shown to be a considerable correction for atomic high-precision experiments. In contrast to this, nuclear polarization effects are of minor importance for Lamb-shift studies in ${ }_{82}^{208} \mathrm{~Pb}$.
\end{abstract}

One of the fascinating aspects of highly ionized atoms is that their study may provide new sensitive tests of quantum electrodynamics in strong external fields. For the analysis of proposed high-precision experiments ${ }^{1-8}$ with one-, two-, or three-electron uranium ${ }_{92}^{238} \mathrm{U}$ a precise knowledge of the electronic spectrum is required. The influence of the finite nuclear size as well as quantum electrodynamical (QED) radiative corrections such as vacuum polarization and self-energy effects on the binding energy of atomic states is well known. ${ }^{9-11}$

At very high precision the additional energy shift of $K$-shell electrons due to nuclear polarization may become relevant. The interaction with internal nuclear degrees of freedom has been extensively studied in the context of muonic atoms, ${ }^{12-14}$ where the resulting energy corrections can be relatively large since binding energies of muons are of the same order as typical nuclear excitation energies. Analogous calculations performed in the case of electronic atoms ${ }^{15,16}$ showed that the predicted energy shifts are much smaller. However, the influence of this contribution increases when the interaction with lowlying nuclear rotational modes is taken into account. One reason is that for heavy elements such as uranium the electronic transition energies become comparable in magnitude with nuclear excitation energies.

Our treatment of the energy shift of strongly bound electrons is based on the introduction of an effective photon propagator containing nuclear-polarization insertions. The effect of nuclear polarization thus appears as part of the radiative corrections to the electron energy. We are particularly concerned with the energy shift of the $1 s_{1 / 2}$ state for a ${ }_{92}^{238} \mathrm{U}$ nucleus, which is the focus of various planed experiments, because it has the highest practically accessible nuclear charge.

Let us first give a brief description of our formal framework. The nuclear charge is described by the electromagnetic current

$$
\hat{j}_{\text {nucl }}^{\mu}(x)=j_{\text {ext }}^{\mu}(\mathbf{x})+\hat{j}_{\text {fluc }}^{\mu}(x),
$$

which consists of a static equilibrium ( $c$ number) part $j_{\text {ext }}^{\mu}$ corresponding to the nucleus in its ground state and a second quantized, time-dependent part $\hat{j}_{\text {fluc }}^{\mu}$ characterizing intrinsic dynamics of the nuclear charge density. The Dirac current $\hat{j}_{e}^{\mu}$ interacts with the electromagnetic field

$$
\hat{A}^{\mu}(x)=A_{\mathrm{ext}}^{\mu}(\mathbf{x})+\hat{A}_{\mathrm{rad}}^{\mu}(x),
$$

where the classical external field $A_{\text {ext }}^{\mu}$ is created by the static nuclear source $j_{\text {ext }}^{\mu}$. The total radiation field $\hat{A}_{\mathrm{rad}}^{\mu}$ is written as the sum of the free photon field $\hat{A}_{\text {free }}^{\mu}$ and a fluctuating field $\hat{A}_{\text {fuc }}^{\mu}$ generated by nuclear-charge fluctuations $\hat{j}_{\text {fluc }}^{\mu}$. The interaction between the electron field and internal nuclear degrees of freedom is described by the interaction Hamiltonian

$$
\hat{H}_{\mathrm{int}}=\int d^{3} x \hat{j}_{e}^{\mu}(x) \hat{A}_{\mu}^{\mathrm{rad}}(x) .
$$

In the Furry picture (bound-state interaction picture) the energy shift of a given electron bound state $|i\rangle$ is expressed by the Tomonaga-Schwinger equation: ${ }^{17}$

$$
e^{-i \Delta E_{i}\left(t_{b}-t_{a}\right)}=\left\langle i\left|T \exp \left(-i \int_{t_{a}}^{t_{b}} d t \hat{H}^{\mathrm{int}}(t)\right)\right| i\right\rangle \text {. }
$$

When performing perturbation expansion one is led to Feynman diagrams with dressed photon lines representing an effective photon propagator defined as the timeordered product

$$
i \mathcal{D}_{\mu \nu}\left(x, x^{\prime}\right)=\left\langle 0\left|T\left[\hat{A}_{\mu}^{\mathrm{rad}}(x) \widehat{A}_{\nu}^{\mathrm{rad}}\left(x^{\prime}\right)\right]\right| 0\right\rangle .
$$

The vacuum expectation value here also implies that the nucleus is considered to be in its ground state. The definition (5) can be written as sum of the free photon propagator $D_{\mu \nu}$ and a polarization correction

$$
\begin{aligned}
\widetilde{D}_{\mu \nu}\left(x, x^{\prime}\right)=\int d^{4} x_{1} \int & d^{4} x_{2} D_{\mu \alpha}\left(x-x_{1}\right) \\
& \times \Pi^{\alpha \beta}\left(x_{1}, x_{2}\right) D_{\beta \nu}\left(x_{2}-x^{\prime}\right),
\end{aligned}
$$

which defines the (reducible) nuclear polarization tensor $\Pi^{\alpha \beta}$. One easily verifies that the polarization tensor is given by the current correlation function

$$
i \Pi^{\alpha \beta}\left(x_{1}, x_{2}\right)=\left\langle 0\left|T\left[\hat{j}_{\text {fluc }}^{\alpha}\left(x_{1}\right) \hat{j}_{\text {fluc }}^{\beta}\left(x_{2}\right)\right]\right| 0\right\rangle .
$$

We will neglect here possible distortions of the nuclear

(c) 1989 The American Physical Society 
excitation spectrum due to the presence of the $K$ electron. Such effects, which occur in muonic atoms in the case of accidental degeneracies are not expected to be important here. Then the time evolution of the nuclear current fluctuation is governed by the nuclear Hamiltonian $\hat{H}_{\text {nucl }}$. Since the nuclear Hamiltonian and the current $\hat{j}_{\text {fluc }}^{\mu}$ are not known from basic principles, one has to apply a nuclear model in order to specify the current and thus the modification $\widetilde{D}_{\mu \nu}$ of the photon propagator. For our purpose here we further neglect the contribution of the nuclear vector current $\widehat{\mathbf{j}}_{\text {fluc }}$ because the velocities associated with nuclear dynamics are mainly nonrelativistic. Accordingly, we deal here only with the longitudinal component $\tilde{D}_{00}$. We are interested in the contribution to the first-order self-energy shift of bound electrons $|i\rangle$ (see Fig. 1) which is given by the expression

$$
\begin{aligned}
\Delta E_{i}=i \alpha \int d^{3} r & d^{3} r^{\prime} \Psi_{i}^{+}(\mathbf{r}) \\
\times & \int d E S_{F}\left(\mathbf{r}, \mathbf{r}^{\prime}, E_{i}-E\right) \\
& \quad \times \widetilde{D}_{00}\left(\mathbf{r}, \mathbf{r}^{\prime}, E\right) \gamma_{o} \Psi_{i}\left(\mathbf{r}^{\prime}\right) .
\end{aligned}
$$

The electron propagator $S_{F}$ satisfies the equation

$$
\left(i \not-e A_{\mathrm{ext}}-m\right) S_{F}\left(\mathbf{r}, \mathbf{r}^{\prime}, t-t^{\prime}\right)=\delta\left(\mathbf{r}-\mathbf{r}^{\prime}\right) \delta\left(t-t^{\prime}\right),
$$

and the wave function $\Psi_{i}$ is a solution of the Dirac equation with external field $A_{\text {ext }}^{\mu}$ and energy eigenvalue $E_{i}$.

In the case of nuclear surface excitations, such as vibrational and rotational modes, the deviation of the nuclear density from the equilibrium density (here taken as
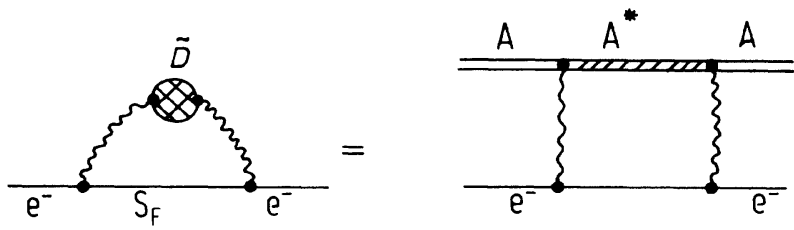

FIG. 1. Modified self-energy as a nuclear-polarization correction.

a homogeneously charged sphere with radius $R_{0}$ ) is given by

$$
\begin{aligned}
\hat{\rho}_{\text {fluc }}(\mathrm{r}, t)= & \rho_{o} R_{o} \delta\left(R_{o}-r\right) \\
& \times \sum_{L M} Y_{L M}^{*}(\hat{r}) \hat{\alpha}_{L M}(t)+\mathcal{O}\left(\alpha^{2}\right), \quad L \geq 2 \\
\hat{\rho}_{\text {fluc }}(\mathbf{r}, t)= & \rho_{o}\left[-3 \Theta\left(R_{o}-r\right)\right. \\
& \left.+R_{o} \delta\left(R_{o}-r\right)\right] Y_{00}(\hat{r}) \hat{\alpha}_{00}(t), \quad L=0
\end{aligned}
$$

where $\hat{\alpha}_{L M}$ denotes the multipole operators of the nuclear surface. The time evolution of the operators $\hat{\alpha}_{L M}$ is governed by the collective Hamiltonian

$$
\hat{H}_{\text {coll }}=\frac{1}{2} \sum_{L M} B_{L} \hat{\alpha}_{L M}^{+} \hat{\dot{\alpha}}_{L M}+\frac{1}{2} \sum_{L M} C_{L} \hat{\alpha}_{L M}^{+} \hat{\alpha}_{L M} .
$$

Evaluating the density correlation function $\Pi^{00}$ of Eq. (7) we obtain the effective photon propagator $\widetilde{D}_{00}$ in Coulomb gauge

$$
\widetilde{D}_{00}\left(\mathbf{r}, \mathbf{r}^{\prime}, E\right)=\sum_{L M L^{\prime} M^{\prime}} \sum_{v} \frac{2 E_{v}}{E^{2}-E_{v}^{2}+i \eta}\left\langle 0\left|\hat{Q}_{L M}\right| v\right\rangle\left\langle v\left|\hat{Q}_{L^{\prime} M^{\prime}}^{+}\right| 0\right\rangle F_{L}(r) F_{L^{\prime}}\left(r^{\prime}\right) Y_{L M^{\prime}}(\hat{r}) Y_{L^{\prime} M^{\prime}}^{*}\left(\hat{r}^{\prime}\right),
$$

where we have introduced the electric multipole operators

$$
\hat{Q}_{L M}=\frac{3}{4 \pi} Z e R_{o}^{L} \hat{\alpha}_{L M}
$$

and $v$ runs over a complete set of collective states. The form of the propagator (11) is rather model independent. Only the radial dependence carried by the functions

$$
\begin{aligned}
& F_{L}(r)=\frac{4 \pi}{R_{o}^{L}(2 L+1)}\left[\begin{array}{l}
\Theta\left(R_{o}-r\right) \frac{r^{L}}{R_{o}^{L+1}} \\
\left.+\Theta\left(r-R_{o}\right) \frac{R_{o}^{L}}{r^{L+1}}\right], L \geq 2
\end{array}\right. \\
& F_{0}(r)=\frac{2 \pi}{R_{o}^{3}} \Theta\left(R_{o}-r\right)\left(1-\frac{r^{2}}{R_{o}^{2}}\right), \quad L=0
\end{aligned}
$$

reflect the sharp surface approximation and the fact that the equilibrium charge density was assumed to be homogeneous and spherical.

In the case of pure rotational excitations the electricmultipole matrix elements have to be evaluated with states $|v\rangle=|L M K\rangle$, whereas one chooses $|v\rangle=|L M\rangle$ in the case of collective surface vibrations. The propagator $\widetilde{D}_{00}$ due to virtual excitation of rotational states thus takes the form

$$
\begin{aligned}
\widetilde{\mathscr{D}}_{00}\left(\mathbf{r}, \mathbf{r}^{\prime}, E\right)=\sum_{L M K} & \frac{2 E_{L K}}{E^{2}-E_{L K}^{2}+i \eta} \\
& \times B(E L ; L K \rightarrow 0) F_{L}(r) F_{L}\left(r^{\prime}\right) \\
& \times Y_{L M}(\hat{r}) Y_{L M}^{*}\left(\hat{r}^{\prime}\right)
\end{aligned}
$$

with the reduced transition probabilities

$$
B(E L ; L K \rightarrow 0)=\frac{\left|\left\langle 0\left\|Q_{L}\right\| L K\right\rangle\right|^{2}}{2 L+1} .
$$

Considering collective vibrations one is led to the analogous expression with corresponding transition probabilities $B(E L ; L \rightarrow 0)$. Let us note that in the same manner the propagator can also be derived for giant resonance excitations. Based on the hydrodynamical model ${ }^{18}$ one obtains somewhat different radial functions $F_{L}(r)$.

We are now in the position to calculate the energy shift (8). For the electron propagator we insert an expansion 
TABLE I. Energy shift of the $1 s_{1 / 2}$ state due to various collective excitations in ${ }_{82}^{208} \mathrm{~Pb}$.

\begin{tabular}{lrc}
\hline \hline$E_{L}(\mathrm{MeV})$ & $B(E L ; L \rightarrow 0)\left(e^{2} b^{L}\right)$ & $\left|\Delta E_{1 s}^{(L)}\right|(\mathrm{meV})$ \\
\hline$E_{0}=13.5$ & 0.199 & 23.58 \\
$E_{1}=13.7$ & 0.072 & 40.30 \\
$E_{2}=12.0$ & 0.106 & 36.40 \\
$E_{2}=4.086$ & 0.060 & 7.89 \\
$E_{3}=2.615$ & 0.096 & 5.65 \\
\hline
\end{tabular}

in terms of eigenfunctions that are solutions of the Dirac equation in the presence of the static external field of a homogeneously charged sphere. In particular, for $K$-shell electrons the resulting energy shift reads as

$$
\begin{aligned}
\Delta E_{1 s}=-\frac{\alpha}{2} \sum_{L K, \kappa} B(E L ; L K \rightarrow 0) M_{1 s, \kappa}^{L}\left(E_{L K}\right), \\
\mathcal{M}_{1 s, \kappa}^{L}\left(E_{L K}\right)=|\kappa| \mid \int_{1}^{\infty} d E \frac{\left|\left\langle 1 s\left|F_{L}\right| E \kappa\right\rangle\right|^{2}}{E-E_{1 s}+E_{L K}} \\
+\sum_{n} \frac{\left|\left\langle 1 s\left|F_{L}\right| n \kappa\right\rangle\right|^{2}}{E_{n \kappa}-E_{1 s}+E_{L K}} \\
\left.+\int_{-\infty}^{-1} d E \frac{\left|\left\langle 1 s\left|F_{L}\right| E \kappa\right\rangle\right|^{2}}{E-E_{1 s}-E_{L K}}\right],
\end{aligned}
$$

with $j=|\kappa|-\frac{1}{2}=L \pm \frac{1}{2}$ for the intermediate states.

The first two terms in $\mathcal{M}_{1 s, \kappa}^{L}$ are equivalent to the usual expression derived in second-order perturbation theory. They mean that a $K$-shell electron can be excited into a higher unoccupied intermediate state by a virtual photon. The third term has to be understood in the chargeconjugated, or time-reversed picture, where a $K$-shell hole can be demoted into the negative-energy continuum (Dirac sea) by emission of an effective photon. This vacuum contribution was not taken into account in previous calculations.

To check our formulation we first considered a candidate for a nuclear vibrator, namely ${ }_{82}^{208} \mathrm{~Pb}$, and calculated the contributions to the energy shift caused by the virtual excitation of the $2^{+}$state at $E_{2}=4.086 \mathrm{MeV}$ and of the low-lying $3^{-}$state at $E_{3}=2.615 \mathrm{MeV}$. We took experimental values for the corresponding reduced transition probabilities. ${ }^{19}$ For completeness we have also calculated the contribution of a monopole vibrational state $E_{0}=13.5 \mathrm{MeV}$ and of the dominant giant dipole resonance at $E_{1}=13.7 \mathrm{MeV}$ with $B(E L)$ values taken from Ref. 20. The energy shift due to the giant quadrupole resonance at $E_{2} \simeq 12 \mathrm{MeV}$ again is based on experimental data obtained recently. ${ }^{21}$ The results are given in Table $I$. They are in a convincing agreement with those obtained by Baur et al., ${ }^{15,16}$ who did not consider octupole $(L=3)$ states.

Turning now to ${ }_{92}^{238} \mathrm{U}$, since we are mostly interested in the effect of low-lying rotational states, we consider the

\begin{tabular}{|c|c|c|c|}
\hline transition & $E(\mathrm{MeV})$ & $B(E L ; L \rightarrow 0)\left(e^{2} b^{L}\right)$ & $\left|\Delta E_{1 s}^{(L)}\right|(\mathrm{meV})$ \\
\hline $2_{\mathrm{gs}}^{+} \rightarrow 0_{\mathrm{gs}}^{+}$ & 0.0411 & 2.38 & 748.4 \\
\hline $2_{\gamma}^{+} \rightarrow 0_{\mathrm{gs}}^{+}$ & 1.0583 & 0.025 & 3.2 \\
\hline $\operatorname{GR}(L=1)$ & 13.0 & 0.135 & 189.7 \\
\hline GR $(L=2)$ & 10.0 & 0.195 & 66.9 \\
\hline
\end{tabular}
following $E 2$ transitions: (a) the transition $2_{\mathrm{gs}}^{+} \rightarrow 0_{\mathrm{gs}}^{+}$
TABLE II. Energy shift of the $1 s_{1 / 2}$ state due to low-lying rotational states and due to giant resonances (GR) in ${ }_{92}^{238} \mathrm{U}$.

within the ground-state band $(K=0)$; (b) the transition from the $2_{\gamma}^{+}$state in the $\gamma$ band $(K=2)$ to the $0_{\mathrm{gs}}^{+}$state of the ground-state band. The corresponding contributions to the energy shift again calculated with experimental $B(E 2)$ values $^{22}$ are displayed in Table II. It also contains the results of a model calculation for the giant dipole and giant quadrupole resonances, respectively.

Obviously the energy shift due to virtual excitation of the $2^{+}$rotational state of the ground-state band is dominant. It is about 2 orders of magnitude larger than the one due to the $E 2$ transition in ${ }_{82}^{208} \mathrm{~Pb}$. This is not surprising in view of the large $B(E 2)$ values. Again one has to add the contribution of giant resonances to the total energy shift. It should also be mentioned that for the types of collective excitations considered here the results do not significantly depend on the explicit form of the radial functions $F_{L}$. We conclude that our results presented here will give at least the right order of magnitude for polarization effects in ${ }_{92}^{238} \mathrm{U}$ due to virtual excitations of lowlying rotational states.

To summarize, we have presented an alternative method to treat nuclear-polarization effects within QED by means of effective-photon propagators. Explicit expressions for the modification of the propagator, i.e., the residual interaction between electrons due to virtual excitation of collective nuclear degrees of freedom have been presented. We considered the energy shift of the $1 s_{1 / 2}$ state and found fair agreement with results obtained earlier for ${ }_{82}^{208} \mathrm{~Pb}$. On the basis of our result we conclude that in ${ }_{92}^{238} \mathrm{U}$ the polarization effects due to low-lying rotational modes will not be negligible in experiments with extremely high precision. Each nuclear excitation contributes additively to the total energy shift. Thus one expects a total energy shift for ${ }_{82}^{208} \mathrm{~Pb}$ of about $0.1 \mathrm{eV}$ while nuclear polarization effects in ${ }_{92}^{238} \mathrm{U}$ yield much larger energy corrections in the order of $1 \mathrm{eV}$, which should be compared with the total $1 \mathrm{~s}$ Lamb shift of about $458 \mathrm{eV}$. The measurement even at the $1 \%$ level of this fundamental and highly nonperturbative quantity is an important goal and would provide a striking test of our understanding of QED in intense Coulomb fields. The fact that uncertainties due to nuclear polarization enters at the $0.2 \%$ level may be considered as of secondary importance. However, we propose to perform future Lamb-shift experiments, aiming at utmost precision tests of quantum electrodynamics, with $\mathrm{Pb}$ ions rather than with uranium.

We thank G. Baur for helpful discussions. 
${ }^{1}$ H. Gould, Nucl. Instrum. Methods B9, 658 (1985).

${ }^{2}$ C. T. Munger and H. Gould, Phys. Rev. Lett. 57, 2927 (1986).

${ }^{3}$ H. F. Beyer, R. D. Deslattes, F. Folkmann, and R. E. LaVilla, J. Phys. B 18, 207 (1985).

${ }^{4}$ P. H. Mokler, Phys. Scr. 36, 715 (1987).

${ }^{5}$ R. D. Deslattes, R. Schuch, and E. Justiniano, Phys. Rev. A 32, 1911 (1985).

${ }^{6}$ E. S. Marmar, J. E. Rice, E. Källne, J. Källne, and R. E. LaVilla, Phys. Rev. A 33, 774 (1986).

${ }^{7}$ A. P. Georgiadis, D. Müller, H.-D Sträter, J. Gassen, P. von Brentano, J. C. Sens, and A. Pape, Phys. Lett. A 115, 108 (1986).

${ }^{8}$ J. P. Briand, P. Indelicato, M. Tavernier, O. Gorceix, D. Liesen, H. F. Beyer, B. Liu, A. Warczak, and J. P. Desclaux, Z. Phys. A 318, 1 (1984).

${ }^{9}$ P. J. Mohr, At. Data Nucl. Data Tables 29, 453 (1983).

${ }^{10}$ W. R. Johnson and G. Soff, At. Data Nucl. Data Tables 33, 405 (1985).

${ }^{11}$ G. Soff and P. J. Mohr, Phys. Rev. A 38, 5066 (1988).

${ }^{12}$ W. Greiner, Z. Phys. 164, 374 (1961).
${ }^{13}$ W. Pieper and W. Greiner, Nucl. Phys. A109, 539 (1968).

${ }^{14}$ E. Borie and G. A. Rinker, Rev. Mod. Phys. 54, 67 (1982).

${ }^{15}$ B. Hoffmann, G. Baur, and J. Speth, Z. Phys. A 315, 57 (1984).

${ }^{16}$ B. Hoffmann, G. Baur, and J. Speth, Z. Phys. A 320, 259 (1985).

${ }^{17}$ S. S. Schweber, An Introduction to Relativistic Quantum Field Theory (Harper \& Row, New York, 1961).

${ }^{18}$ J. M. Eisenberg and W. Greiner, Nuclear Models (NorthHolland, Amsterdam, 1970).

${ }^{19}$ A. M. R. Joye, A. M. Baxter, M. P. Fewell, D. C. Kean, and R. H. Spear, Phys. Rev. Lett. 38, 807 (1977).

${ }^{20}$ G. A. Rinker and J. Speth, Nucl. Phys. A306, 360 (1978).

${ }^{21}$ J. Barrette, N. Alamanos, F. Auger, B. Fernandez, A. Gillibert, D. J. Horen, J. R. Beene, F. E. Bertrand, R. L. Auble, B. L. Burks, J. Gomez del Campo, M. L. Halpert, R. O. Sayer, W. Mittig, Y. Schutz, B. Haas, and J. P. Vivien, Phys. Lett. B 209, 182 (1988).

${ }^{22}$ V. P. Varshney, K. K. Gupta, A. K. Chaubey, and D. K. Gupta, Can. J. Phys. 60, 1461 (1982). 